\title{
Skeletal muscle lipid quantification in lean and diabetic subjects using in vivo proton MR spectroscopy
}

\author{
Sunil K Valaparla1, Goldie R E Boone ${ }^{1}$, Erika M Ripley', Daniele Giuseppe², Timothy Q Duong1, \\ Muhammad Abdul-Ghani², Geoffrey D Clarke ${ }^{1}$ \\ ${ }^{1}$ Research Imaging Institute, UT Health Science Center at San Antonio, San Antonio, TX, USA. \\ ${ }^{2}$ Diabetes Division, UT Health Science Center at San Antonio, San Antonio, TX, USA.
}

Received March 19, 2014; Published Online April 08, 2014

[Presented at the Young Investigator's Symposium at the 2014 Annual Meeting of

Southwest Chapter of American Association of Physicists in Medicine (AAPM) in San Antonio, Texas, USA]

\section{Conference Proceeding}

\begin{abstract}
Purpose: To quantify and compare the intramyocellular (IMCL), extramyocellular (EMCL) lipids and total fat fraction in human vastus lateralis muscle between lean and type 2 diabetic (T2DM) subjects using long echo time (TE) STEAM proton MR spectroscopy ( $\left.{ }^{1} \mathrm{H}-\mathrm{MRS}\right)$.

Methods: ${ }^{1} \mathrm{H}-\mathrm{MRS}$ using single voxel $\left(15 \times 15 \times 15 \mathrm{~mm}^{3}\right)$ stimulated acquisition mode (STEAM) was performed in right vastus lateralis $\mathrm{m}$. on 10 lean controls (age: $28.3 \pm 3.94$ yo, BMI: $24.25 \pm 3.20 \mathrm{~kg} / \mathrm{m}^{2}$ ) and 7 type 2 diabetic (age: 54.28 \pm 6.42 yo, BMI: $31.34 \pm 3.13 \mathrm{~kg} / \mathrm{m}^{2}$ ) subjects with Siemens 3T MRI and a four-channel flex coil. Unsuppressed water spectra $(\mathrm{NSA}=16)$ with $\mathrm{TR} / \mathrm{TE}=3000 / 30 \mathrm{~ms}, \mathrm{TM}=10 \mathrm{~ms} \mathrm{BW}=$ $2000 \mathrm{~Hz}$, and water-suppressed spectra (NSA = 128) with $\mathrm{TR} / \mathrm{TE}=3000 / 270 \mathrm{~ms}, \mathrm{TM}=10 \mathrm{~ms}$, fixed water suppression $\mathrm{BW}=50 \mathrm{~Hz}$ were acquired. Spectral intensity ratios of IMCL-CH2, EMCL-CH2 and total lipid (IMCL + EMCL) with unsuppressed water signal (W) were converted into absolute concentrations expressed in $\mathrm{mmol} / \mathrm{kg}$. Fat fraction $(100 \times$ $\mathrm{F} /(\mathrm{W}+\mathrm{F}))$ was also calculated, where $\mathrm{F}$ includes the signal intensities of IMCL and EMCL methylene $\left(\mathrm{CH}_{2}\right)$ n, peaks only.
\end{abstract}

Presenting author: Sunil Kumar Valaparla; Research Imaging Institute, UT Health Science Center at San Antonio, San Antonio, TX, USA.

Cite this article as:

Valaparla SK, Boone GRE, Ripley EM, Giuseppe D, Duong TQ Abdul-Ghani M, Clarke GD. Skeletal muscle lipid quantification in lean and diabetic subjects using in vivo proton MR spectroscopy. Int J Cancer Ther Oncol 2014; 2(2):020239. DOI: $10.14319 / \mathrm{ijcto} 0202.39$
Results: Comparison of IMCL (controls: $11.70 \pm 6.7$, T2DM: $21.74 \pm 10.2, \mathrm{p}<0.01$ ), EMCL (controls: $22.89 \pm 18.42$, T2DM: $77.21 \pm 33.4, \mathrm{p}<0.001)$ and total lipid $(64.35$ $\mathrm{mmol} / \mathrm{kg}$ less in controls, $\mathrm{p}<0.001$ ) showed statistical significance using two-tailed student's t-test. Mean fat fraction (\%) exhibited considerable inter-individual variability for controls (3.14 \pm 2.09 ; range: $1.34-7.04)$ and T2DM $(9.34 \pm 2.88$; range: $4.15-13.67)$ and deemed significant $(\mathrm{p}<0.05)$.

Conclusion: Single voxel STEAM ${ }^{1} \mathrm{H}-\mathrm{MRS}$ at long TE provides a robust non-invasive method for characterizing lipids within localized muscle regions, with well-resolved IMCL/EMCL peak separation. Regional lipid estimate and fat fraction in vastus lateralis muscle is significantly different in T2DM compared to normal lean controls.

Impact: Single-voxel proton MR spectroscopy $\left({ }^{1} \mathrm{H}-\mathrm{MRS}\right)$ with STEAM at long echo time (TE = $270 \mathrm{~ms}$ ) provides a robust non-invasive method for characterizing muscle lipids in vivo. This method offers rapid and convenient acquisition of high -resolution spectra with improved baseline and better lipid peak separation in skeletal muscle and allows for fat quantification with superior sensitivity and dynamic range over other MR imaging methods. This study quantified IMCL, EMCL and fat fraction using ${ }^{1} \mathrm{H}-\mathrm{MRS}$ from vastus lateralis $m$., predominantly utilized in $e x$ vivo physiological studies for histochemical evaluation of muscle lipid metabolism in diabetes and obesity. The method described can be combined with biochemical assays to provide insights on excess skeletal muscle fat interference with insulin signaling in obese and diabetic states in longitudinal clinical research studies 
Key Results:

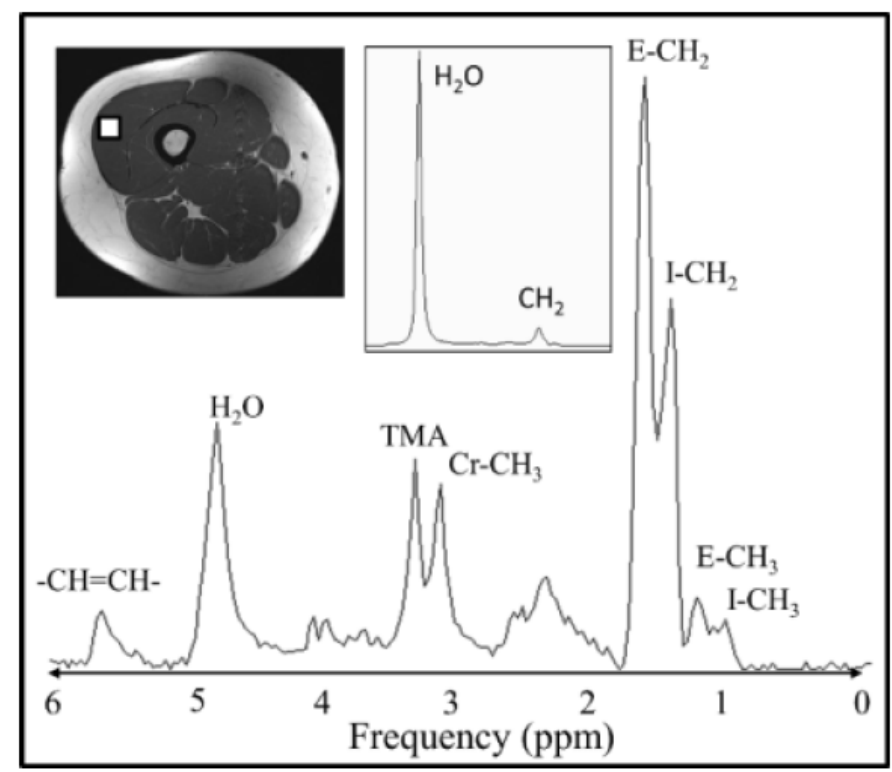

FIG. 1: STEAM ${ }^{1} \mathrm{H}-\mathrm{MRS}$ voxel of interest (VOI $3.5 \mathrm{~mL}$ ) placement for vastus lateralis muscle fat quantification on the T1-weighted axial image of the right thigh midsection. Representative water suppressed (WS) lipid MR spectrum acquired at TE $=270 \mathrm{~ms}$, showing clear separation between methylene IMCL-CH 2 and EMCL- $\mathrm{CH}_{2}$ peaks. Signal intensities from methyl (-CH3) components, $\mathrm{Cr}_{3} \mathrm{CH}_{3}$, TMA and $\mathrm{CH}=\mathrm{CH}$ fat peak at $5.4 \mathrm{ppm}$ are also observed with superior resolution and SNR. Sub-figure shows water reference (WR) spectra obtained at TE $=30 \mathrm{~ms}$. Ratio of (IMCL-CH2 + EMCL-CH2) lipid peak area from WS and $\mathrm{H}_{2} \mathrm{O}$ peak from WR, corrected for both $\mathrm{T} 1$ and $\mathrm{T} 2$ decays provided the fat fraction within the VOI.

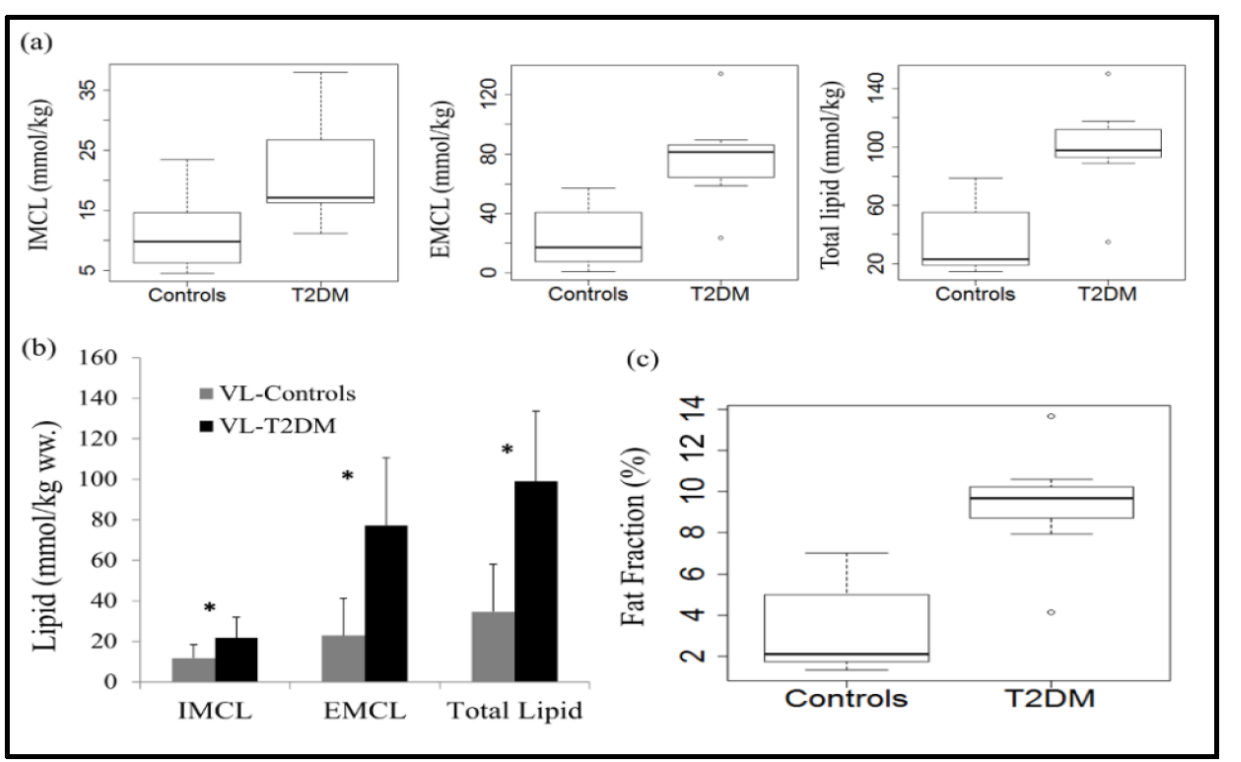

FIG. 2: a) Box-whisker plots of IMCL, EMCL and total lipids (converted into absolute concentrations (mmol/kg) from spectral intensity ratios) measured between lean controls and type 2 diabetic (T2DM) showed considerable variability and statistical significant at $\mathrm{p}<0.001$. Horizontal line in each box is the median. Lower and upper ends of each box are limits of first and third quartiles, and height of each box is interquartile range. Vertical lines extending above and below each box end on data points farthest from mean but within distance of 1.5 times interquartile range from end of each box. Data points that are outliers are located between 1.5 and three times the interquartile range from end of each box. b) Bar plots (mean \pm sd) of IMCL, EMCL and total lipids in the vastus lateralis muscle between lean and T2DM ( $\left.{ }^{*} \mathrm{p}<0.05\right)$ c) Muscle fat fraction (\%) measured by ${ }^{1} \mathrm{H}-\mathrm{MRS}$ in lean controls (mean \pm sd: $3.14 \pm 2.09$; range: $\left.1.34-7.04\right)$ and T2DM $(9.34 \pm 2.88$; range: $4.15-13.67)$. 